\title{
ON QUOTIENTS OF GENERALIZED EUCLIDEAN GROUP RINGS
}

\author{
LUC GUYOT
}

\begin{abstract}
Let $R=\mathbb{Z}[C]$ be the integral group ring of a finite cyclic group $C$. Dennis et al. proved that $R$ is a generalized Euclidean ring in the sense of P. M. Cohn, i.e., $\mathrm{SL}_{n}(R)$ is generated by the elementary matrices for all $n$. We prove that every proper quotient of $R$ is also a generalized Euclidean ring.
\end{abstract}

\section{INTRODUCTION}

Let $R$ be an associative ring with identity. For $n \geq 2$, we define $\mathrm{E}_{n}(R)$ as the subgroup of $\mathrm{GL}_{n}(R)$ generated by the elementary matrices, i.e., the matrices which differ from the identity by a single off-diagonal element. The ring $R$ is termed a $\mathrm{GE}_{n}$-ring if $\mathrm{GL}_{n}(R)=\mathrm{D}_{n}(R) \mathrm{E}_{n}(R)$, where $\mathrm{D}_{n}(R)$ denotes the subgroup of invertible diagonal matrices. Following P. M. Cohn, we term $R$ a generalized Euclidean ring, or concisely a GE-ring, if $R$ is a $\mathrm{GE}_{n}$-ring for every $n \geq 2$, a property notoriously shared by Euclidean rings. If $R$ is commutative, Whitehead's lemma implies that $\mathrm{D}_{n}(R) \cap \mathrm{SL}_{n}(R) \subset \mathrm{E}_{n}(R)$. Therefore the property $\mathrm{GE}_{n}$ is equivalent to $\mathrm{SL}_{n}(R)=\mathrm{E}_{n}(R)$ in this case. Let $I$ be an ideal of a commutative ring $R$ and let $\varphi_{n}$ be the natural map $\mathrm{SL}_{n}(R) \rightarrow \mathrm{SL}_{n}(R / I)$. The natural map $\mathrm{E}_{n}(R) \rightarrow \mathrm{E}_{n}(R / I)$ is surjective and if $\varphi_{n}$ is surjective too, then $R / I$ is certainly a $\mathrm{GE}_{n}$-ring whenever $R$ is. But $\varphi_{n}$ can fail to be surjective for all $n \geq 2$ as is the case for $R=\mathbb{R}[X, Y]$ and $I$ the principal ideal generated by $X^{2}+Y^{2}-1$ (see [Mil71, Example 13.5] and [Lam06, Proposition I.8.12 and Theorem VI.4.5]). Thus the inheritance of property $\mathrm{GE}_{n}$ by homomorphic images is not automatic. In the previous example $R$ is a $\mathrm{GE}_{3}$-ring whereas $R / I$ is not a $\mathrm{GE}_{n}$-ring for any $n \geq 2$. We can actually produce a non-commutative GE-ring having a non- $\mathrm{GE}_{2}$ quotient. Indeed, a non-commutative free associative algebra over a field $k$ is a GE-ring

Date: November 8, 2016.

2010 Mathematics Subject Classification. Primary 13F07, Secondary 16S34.

Key words and phrases. group ring; quotient; generalized Euclidean ring; quasi-Euclidean ring; elementary matrix; unimodular row; Nielsen equivalence; metabelian group. 
LUC GUYOT

and maps homomorphically onto a free commutative algebra over $k$ which is not a $\mathrm{GE}_{2}$-ring by [Coh66, Theorem 3.4 and Proposition 7.3].

By contrast, the class of commutative GE-rings is known to be stable under taking quotients by the ideals contained in the Jacobson radical Gel77, Proposition 5]. In addition, the class of quasi-Euclidean rings, i.e., the GErings which are moreover Hermite rings in the sense of Kaplansky Kap49, is stable under taking quotients by any ideals [AJLL14.

The integral group ring $\mathbb{Z}[C]$ of a finite cyclic group $C$ was shown to be a GE-ring in [DMV84. If $C$ is not trivial, then $\mathbb{Z}[C]$ has a non-principal ideal, and hence is not quasi-Euclidean. The purpose of this article is to establish

Theorem A. Let $C$ be a finite cyclic group. Then every homomorphic image of $\mathbb{Z}[C]$ is a GE-ring.

Our result has some bearing in combinatorial group theory. Let $R$ be quotient of $\mathbb{Z}[C]$ and let $M$ be a finitely generated free $R$-module with $C$ a finite cyclic group. Then the image of $C$ in $R$ is a subgroup of the unit group of $R$ so that $C$ acts naturally on $M$ by automorphisms. Theorem $\mathrm{A}$ allows us to show that the semi-direct product $G=M \rtimes C$ has only one Nielsen class of generating $k$-tuples for every $k \geq \operatorname{rk}(G)+1$, where $\operatorname{rk}(G)$ denotes the minimal number of generators of $G$ [Guy16, Corollary 4].

Our proof of Theorem $\mathrm{A}$ reuses extensively the techniques developed in [DMV84. Lemma 4 below compiles all criteria from the latter article which render induction possible. The proof of these criteria is faithful to the original. We provide it for the reader's convenience. A notable difference in our case is that we need to extend the induction basis to the integer ring $\mathbb{Z}\left[\zeta_{d}\right]$ of the cyclotomic field $\mathbb{Q}\left(\zeta_{d}\right)\left(\zeta_{d}=e^{\frac{2 i \pi}{d}}\right)$ for all positive rational integers $d \notin\{1,2,3,4,6\}$. For this we can rely on Vas72. The basis of our induction also comprises a set of 31 small quotients of $\mathbb{Z}[X]$ parametrized by the non-empty subsets of $\{1,2,3,4,6\}$. These quotients can in turn be handled by induction, still using Lemma 4 .

Acknowledgments. The author thanks Pierre de la Harpe and Tatiana SmirnovaNagnibeda for encouragements and comments made on preliminary versions of this paper.

\section{ProOF}

Our first step consists in the following observation: we only need to prove that $\mathrm{GE}_{2}$ holds for the homomorphic images of $\mathbb{Z}[C]$. This is the assertion $(i i)$ of this first lemma which collects elementary facts for later use: 
Lemma 1. Let $R$ be a commutative ring with identity. Then the following assertions hold:

(i) If the stable rank of $R$ is 1 , then $R$ is a GE-ring.

(ii) If the stable rank of $R$ is at most 2 (e.g., the Krull dimension of $R$ is at most 1$)$, then $R$ is a GE-ring if and only if it is a $\mathrm{GE}_{2}$-ring.

(iii) $R$ is a $\mathrm{GE}_{2}$-ring if and only if $\mathrm{E}_{2}(R)$ acts transitively on the set

$$
\mathrm{Um}_{2}(R)=\left\{(r, s) \in R^{2} \mid r R+s R=R\right\}
$$

of unimodular pairs of $R$.

A semilocal ring has stable rank 1 [Bas64, Corollary 6.5]. As a result semilocal rings, and Artinian rings in particular, are GE-rings. All rings considered in the proof of Theorem $\mathrm{A}$ are quotients of $\mathbb{Z}[C]$ and hence have stable rank at most 2 [MR87, Corollary 6.7.4].

Our second step consists in a reduction to quotients of $\mathbb{Z}[C]$ of a special kind. To this end, we will need the following stability properties:

Lemma 2. Let $R$ be a commutative ring with identity. Then the following assertions hold:

(i) Let $J$ be an ideal contained in the Jacobson radical of $R$. Then $R$ is a GE-ring if and only if $R / J$ is a GE-ring [Gel77, Proposition 5].

(ii) If $R / I$ is a GE-ring for some finite ideal $I$ of $R$, then $R$ is a GE-ring.

Proof. ( $i$ ) See aforementioned reference. (ii). Let $n \geq 2$ and let $A \in \mathrm{SL}_{n}(R)$. As $R / I$ is a GE-ring, we can find $E \in \mathrm{E}_{n}(R)$ such that $B=A E^{-1}$ lies in $\mathrm{SL}_{n}(R, I) \doteqdot \operatorname{ker}\left(\mathrm{SL}_{n}(R) \rightarrow \mathrm{SL}_{n}(R / I)\right)$. The coefficients of $B$ lie in the subring $K$ of $R$ generated by $I$ and $1_{R}$, the identity of $R$. Therefore it suffices to show that $K$ is a GE-ring. If the prime field of $R$ is finite, then $K$ is finite and hence Artinian. As $K$ is a GE-ring in this case, we can assume now that the prime field of $R$ is $\mathbb{Z}$. We have then $\mathbb{Z} 1_{R} \cap I=0$, which implies that the additive group of $K$ is isomorphic to $\mathbb{Z} \times I$. Let $e$ be the exponent of the additive group of $I$. Then $K / e K$ is finite and hence a GE-ring. We can find $E^{\prime} \in \mathrm{E}_{n}(K)$ such that $C=B E^{\prime-1}$ lies in $\mathrm{SL}_{n}(K, e K) \subset \mathrm{SL}_{n}\left(\mathbb{Z} 1_{R}\right)$. As $\mathbb{Z} 1_{R} \simeq \mathbb{Z}$ is Euclidean, we have $C \in \mathrm{E}_{n}\left(\mathbb{Z} 1_{R}\right)$, which completes the proof.

We now introduce the special $\mathrm{GE}_{2}$-quotients of $\mathbb{Z}[X]$ to which the study of $\mathbb{Z}[C]$ can be reduced. Given a rational integer $d>0$ we let $\lambda_{d}: \mathbb{Z}[X] \rightarrow \mathbb{Z}\left[\zeta_{d}\right]$ be the ring homomorphism induced by the map $X \mapsto \zeta_{d}$. Given a set $\mathcal{D}$ of positive rational integers, we define $\lambda_{\mathcal{D}}: \mathbb{Z}[X] \rightarrow \prod_{d \in \mathcal{D}} \mathbb{Z}\left[\zeta_{d}\right]$ by $\lambda_{\mathcal{D}}=\prod_{d \in \mathcal{D}} \lambda_{d}$ and set $\mathcal{O}(\mathcal{D})=\lambda_{\mathcal{D}}(\mathbb{Z}[X])$.

Lemma 3. Let $\mathcal{D}$ be a finite and non-empty set of positive rational integers. Then $\mathcal{O}(\mathcal{D})$ is a $\mathrm{GE}_{2}$-ring. 
We postpone the proof of Lemma 3 and prove the theorem first.

Proof of Theorem $\mathbb{A}$. Set $n=|C|$ and let $R$ be an homomorphic image of $\mathbb{Z}[X]$. There exists an ideal $I$ of $\mathbb{Z}[X]$ containing $X^{n}-1$ and such that $R=\mathbb{Z}[X] / I$. If $R$ is finite, then $R$ is Artinian and hence a GE-ring. Thus we can assume that $R$ is infinite. By Lemma $2, i$ we can also assume that $R$ is reduced. Since $R$ is Noetherian, the ideal $I$ is the intersection of finitely many prime ideals of $\mathbb{Z}[X]$. Those ideals contain $X^{n}-1=\prod_{d \mid n} \Phi_{d}(X)$, therefore they are either principal ideals generated by cyclotomic polynomials or maximal ideals. In the latter case the index in $R$ is necessarily finite. Consider then the finite and non-empty set $\mathcal{D}$ of all positive rational integers such that $I \subset \Phi_{d}(X) \mathbb{Z}[X]$ and let $\pi: \mathbb{Z}[X] \rightarrow R$ be the natural ring epimorphism. Using the quotients of $\mathbb{Z}[X]$ by the prime ideals containing $I$, we easily see that there is a finite direct sum $K$ of finite fields, an injective ring homomorphism $\lambda: R \rightarrow \prod_{d \in \mathcal{D}} \mathbb{Z}\left[\zeta_{d}\right] \times K$ and a surjective ring homomorphism $\kappa: R \rightarrow K$ such that $\lambda \circ \pi=\lambda_{\mathcal{D}} \times \kappa$. Then the projection $\prod_{d \in \mathcal{D}} \mathbb{Z}\left[\zeta_{d}\right] \times K \rightarrow \prod_{d \in \mathcal{D}} \mathbb{Z}\left[\zeta_{d}\right]$ induces a ring epimorphism from $R$ onto $\mathcal{O}(\mathcal{D})$ whose kernel is $R \cap(\{0\} \times K), R$ being identified with $\lambda(R)$. Applying Lemma 2 , ii yields the conclusion.

The remainder of this section is devoted to the proof of Lemma 3 .

Lemma 4. Let $\mathcal{D}$ be a finite set of positive rational integers with at least two elements. Let $e \in \mathcal{D}$ and set $\eta_{e}=\prod_{d \in \mathcal{D} \backslash\{e\}} \Phi_{d}\left(\zeta_{e}\right)$. Assume that $\mathcal{O}(\mathcal{D} \backslash\{e\})$ is $a \mathrm{GE}_{2}$-ring and that at least one of the following holds:

(i) $e \notin\{1,2,3,4,6\}$ and $\frac{d}{e}$ and is not a positive power of a prime for any $d \in \mathcal{D} \backslash\{e\}$.

(ii) $e \in\{1,2,3,4,6\}$ and $\eta_{e} \in\left(\mathbb{Z}\left[\zeta_{e}\right]\right)^{\times}$.

(iii) $e \in\{1,2\}$ and $\eta_{e}= \pm 3$.

(iv) $e \in\{1,2,3,6\}$ and $\eta_{e} \mathbb{Z}\left[\zeta_{e}\right]=2 \mathbb{Z}\left[\zeta_{e}\right]$.

$(v) e \in\{3,4,6\}$ and $\eta_{e} \mathbb{Z}\left[\zeta_{e}\right]=\left(1-\zeta_{e}\right)^{k} \mathbb{Z}\left[\zeta_{e}\right]$ for some $k \in\{1,2\}$.

Then $\mathcal{O}(\mathcal{D})$ is a $\mathrm{GE}_{2}$-ring.

Proof. Assertion $(i)$ is [DMV84, Lemma 3.5]. For the remaining assertions we identify $\mathcal{O}(\mathcal{D})$ with its image in $\mathcal{O}(\mathcal{D} \backslash\{e\}) \times \mathbb{Z}\left[\zeta_{e}\right]$ and let $\mathbf{r}=\left(\left(\begin{array}{l}a \\ c\end{array}\right),\left(\begin{array}{l}b \\ d\end{array}\right)\right) \in$ $\mathrm{Um}_{2}(R)$. Since the projection $\operatorname{pr}_{e}: \mathcal{O}(\mathcal{D}) \rightarrow \mathcal{O}(\mathcal{D} \backslash\{e\})$ is surjective and $\mathcal{O}(\mathcal{D} \backslash\{e\})$ is a $\mathrm{GE}_{2}$-ring, after multiplication by some $E \in \mathrm{E}_{2}(\mathcal{O}(\mathcal{D}))$ we can assume that

$$
a=1, b=0
$$


As $\operatorname{ker}\left(\operatorname{pr}_{e}\right)$ maps onto $\eta_{e} \mathbb{Z}\left[\zeta_{e}\right]$ via the projection $\mathcal{O}(\mathcal{D}) \rightarrow \mathbb{Z}\left[\zeta_{e}\right]$, we have

$$
\begin{array}{r}
c-1, d \in \eta_{e} \mathbb{Z}\left[\zeta_{e}\right] \\
\mathcal{O}(\mathcal{D}) \cap\left(\{0\} \times \mathbb{Z}\left[\zeta_{e}\right]\right)=\{0\} \times \eta_{e} \mathbb{Z}\left[\zeta_{e}\right] .
\end{array}
$$

Conditions (11) and (2) remain valid after multiplication by any product of matrices in

$$
\left(\begin{array}{cc}
1 & \{0\} \times \eta_{e} \mathbb{Z}\left[\zeta_{e}\right] \\
0 & 1
\end{array}\right) \bigcup\left(\begin{array}{cc}
1 & 0 \\
\mathcal{O}(\mathcal{D}) & 1
\end{array}\right)
$$

The projection of these of matrices to the $\mathbb{Z}\left[\zeta_{e}\right]$ coordinate is

$$
\left(\begin{array}{cc}
1 & \eta_{e} \mathbb{Z}\left[\zeta_{e}\right] \\
0 & 1
\end{array}\right) \bigcup\left(\begin{array}{cc}
1 & 0 \\
\mathbb{Z}\left[\zeta_{e}\right] & 1
\end{array}\right)
$$

If $(i i)$ holds then matrices of the form (3) generate $\mathrm{E}_{2}\left(\mathbb{Z}\left[\zeta_{e}\right]\right)$. As $\mathbb{Z}\left[\zeta_{e}\right]$ is a Euclidean ring for $e \in\{1,2,3,4,6\}$ such matrices can therefore be used to reduce $(c, d)$ to $(1,0)$, reducing thus $\mathbf{r}$ to $(1,0)$.

If either $(i i i)$ or $(i v)$ holds, then [DMV84, Lemma 4.1] applies, so that multiplication by matrices in (3) can reduce either $c$ or $d$ to 0 . Since $c-1 \in$ $\eta_{e} \mathbb{Z}\left[\zeta_{e}\right]$, it must be $d$. Assuming now that $d=0$, the unimodularity of $(c, d)$ yields

$$
c \in\left(1+\eta_{e} \mathbb{Z}\left[\zeta_{e}\right]\right) \cap\left(\mathbb{Z}\left[\zeta_{e}\right]\right)^{\times} \subset\{ \pm 1\} .
$$

If $c=-1$, then $\eta_{e} \mathbb{Z}\left[\zeta_{e}\right]=2 \mathbb{Z}\left[\zeta_{e}\right]$ must hold and the identity

$$
\left(\begin{array}{ll}
c & d
\end{array}\right)\left(\begin{array}{ll}
1 & 2 \\
0 & 1
\end{array}\right)\left(\begin{array}{cc}
1 & 0 \\
-1 & 1
\end{array}\right)\left(\begin{array}{ll}
1 & 2 \\
0 & 1
\end{array}\right)=\left(\begin{array}{ll}
1 & 0
\end{array}\right)
$$

shows that $(c, d)$ can be reduced to $(1,0)$ using matrices in (3).

If $(v)$ holds then $\left(\mathbb{Z}\left[\zeta_{e}\right], 1-\zeta_{e}\right)$ is a Euclidean pair with respect to the complex modulus, so that [DMV84, Lemma 4.2] applies and leads to the desired conclusion.

Proof of Lemma 3. We apply inductively Lemma 4, $i$ by choosing at each step the largest member $e$ of $\mathcal{D}$ which is not in $S=\{1,2,3,4,6\}$. In this way $\mathcal{D}$ is reduced to either a subset of $S$ or a singleton $\{d\}$ with $d \notin S$. In the latter case, $\mathcal{O}(\mathcal{D})=\mathcal{O}(\{d\})=\mathbb{Z}\left[\zeta_{d}\right]$ is a $\mathrm{GE}_{2}$-ring by Vas72. We also observe that $\mathcal{O}(\{d\})$ is a $\mathrm{GE}_{2}$-ring for all $d$ in $S$. Indeed, the rings $\mathbb{Z}, \mathbb{Z}[i]$ and $\mathbb{Z}\left[\zeta_{3}\right]$ are Euclidean rings with respect to the complex modulus. Applying assertions $(i i)$ to $(i v)$ of Lemma 4, we can prove the result inductively for all pairs, triples and quadruples of elements of $S$, except the quadruple $\{1,2,3,6\}$ for which we apply [DMV84, Proposition 5.1]. Eventually, we apply Lemma 4. $v$ (or equivalently [DMV84, Proposition 5.10]) to prove the result for $\mathcal{D}=S$. 


\section{REFERENCES}

[AJLL14] Adel Alahmadi, S. K. Jain, T. Y. Lam, and A. Leroy. Euclidean pairs and quasiEuclidean rings. J. Algebra, 406:154-170, 2014.

[Bas64] H. Bass. K-theory and stable algebra. Inst. Hautes Études Sci. Publ. Math., (22):5-60, 1964.

[Coh66] P. M. Cohn. On the structure of the $\mathrm{GL}_{2}$ of a ring. Inst. Hautes Études Sci. Publ. Math., (30):5-53, 1966.

[DMV84] Keith Dennis, Bruce Magurn, and Leonid Vaserstein. Generalized Euclidean group rings. J. Reine Angew. Math., 351:113-128, 1984.

[Gel77] Susan C. Geller. On the $G E_{n}$ of a ring. Illinois J. Math., 21(1):109-112, 1977.

[Guy16] L. Guyot. Generators of split extensions of Abelian groups by cyclic groups. Preprint, arXiv: [math.GR], April 2016.

[Kap49] Irving Kaplansky. Elementary divisors and modules. Trans. Amer. Math. Soc., 66:464-491, 1949.

[Lam06] T. Y. Lam. Serre's problem on projective modules. Springer Monographs in Mathematics. Springer-Verlag, Berlin, 2006.

[Mil71] John Milnor. Introduction to algebraic K-theory. Princeton University Press, Princeton, N.J.; University of Tokyo Press, Tokyo, 1971. Annals of Mathematics Studies, No. 72.

[MR87] J. C. McConnell and J. C. Robson. Noncommutative Noetherian rings. Pure and Applied Mathematics (New York). John Wiley \& Sons, Ltd., Chichester, 1987. With the cooperation of L. W. Small, A Wiley-Interscience Publication.

[Vas72] L. N. Vaseršteĭn. The group $S L_{2}$ over Dedekind rings of arithmetic type. Mat. Sb. (N.S.), 89(131):313-322, 351, 1972.

EPFL EnT CBS BBP/HBP. Campus Biotech. B1 Building, Chemin des mines, 9, Geneva 1202, Switzerland

E-mail address: luc.guyot@epfl.ch 